\title{
Brown Dwarf Model Atmospheres Based on Multi-Dimensional Radiation Hydrodynamics
}

\author{
France Allard and Bernd Freytag \\ Centre de Recherche Astrophysique de Lyon, \\ UMR 5574: CNRS, Université de Lyon, École Normale Supérieure de Lyon, \\ 46 allée d'Italie, F-69364 Lyon Cedex 07, France \\ email: fallard@ens-lyon.fr
}

\begin{abstract}
The atmospheres of Brown Dwarfs (BDs) are the site of molecular opacities and cloud formation, and control their cooling rate, radius and brightness evolution. Brown dwarfs evolve from stellar-like properties (magnetic activity, spots, flares, mass loss) to planet-like properties (electron degeneracy of the interior, cloud formation, dynamical molecular transport) while retaining, due to their fully convective interior, larger rotational velocities $(\leqslant 30 \mathrm{~km} / \mathrm{s}$ i.e. $\mathrm{P}<4$ hrs versus $11 \mathrm{hrs}$ for Jupiter). Model atmospheres treating all this complexity are therefore essential to understand the evolution properties, and to interpret the observations of these objects. While the pure gas-phase based NextGen model atmospheres (Allard et al. 1997, Hauschildt et al. 1999) have allowed the understanding of the several populations of Very Low Mass Stars (VLMs), the AMES-Dusty models (Allard et al. 2001) based on equilibrium chemistry have reproduced some near-IR photometric properties of $\mathrm{M}$ and L-type brown dwarfs, and played a key role in the determination of the mass of brown dwarfs and Planetary Mass Objects (PMOs) in the eld and in young stellar clusters. In this paper, we present a new model atmosphere grid for VLMs, BDs, PMOs named BT-Settl, which includes a cloud model and dynamical molecular transport based on mixing information from 2D Radiation Hydrodynamic (RHD) simulations (Freytag et al. 2009). We also present the status of our 3D RHD simulations including rotation (Coriolis forces) of a cube on the surface of a brown dwarf. The BT-Settl model atmosphere grid will be available shortly via the Phoenix web simulator (http://phoenix.ens-lyon.fr/simulator/).
\end{abstract}

Keywords. stellar atmospheres, brown dwarfs, very low mass stars, radiation hydrodynamics

\section{References}

Allard, F., Hauschildt, P. H., Alexander, D. R., \& Starrfield, S. 1997, ARA\&A, 35, 137

Allard, F. et al. 2001, ApJ, 556, 357

Freytag, B., Allard, F., Ludwig, H.-G., Homeier, D., \& Steffen, M. 2009, A\&A, in press

Hauschildt, P. H., Allard, F., \& Baron, E. 1999, ApJ, 512, 377 